\title{
POST-PEAK RESPONSE AUTOMATIC SOLUTIONS IN STRUCTURAL ENGINEERING PROBLEMS - A REVIEW
}

\author{
Husain K. Jarallah
}

Assistant Professor, Department of Civil Engineering, College of Engineering, Mustansiriyah University, Baghdad, Iraq. Email: khalfdce@uomustansiriyah.edu.iq

\section{HTTPS://DOI.ORG/10.30572/2018/KJE/120404}

\begin{abstract}
The ill-condition of stiffness matrix at the unstable region for example at the strain-softening region, the load control method will not be valid to give the solution therefore the displacement control method is essential to use. The nonlinear solution methods under the umbrella of Newton's family that can be used to capture the changing in the stiffness matrix solution at different steps of load control. The Newton's method has disadvantage that cannot get the solution at unstable region therefore the solution will be termination when the any values of diagonal element of global stiffness matrix becomes zero or no positive value, these issue has been solved by using arc-length method in the nonlinear analysis. A scientific review of literature has been made to pinpoint the difficulties faced in the prediction of complete load-displacement response of structures and techniques available to overcome the same. Displacement control methods have been reviewed with emphases on the arc length methods which can be integrated with the traditional finite element method.
\end{abstract}

KEYWORDS: Displacement control, Nonlinear Analysis, Post peak response, IncrementalIteration Method, Snap-through. 


\section{INTRODUCTION}

The complete nonlinear solution path of structure including limit points and beyond of this point is need to check and track.. Newton-Raphson method is called Load controlled method, it is unsuccessful near the limit point for geometrically and material nonlinearity problem, therefore the displacement control method is required for the solution beyond the ultimate point of the load-displacement relationship. The design of structure can be improved when the post-critical behavior is monitoring. A typical example is the most economical design and imperfection sensitivity of certain structures, which in general is directly associated with the post-critical response. The most economical design approach requires an analytical method, which aims at the complete description of the static response of structures. Hence, one has to adopt a suitable numerical method to trace nonlinear sub-critical and post-critical equilibrium paths and to obtain detailed information about the position and nature of instability phenomena. The conventional method of the nonlinear solution consists of the use of an increment loading when procedure combined with incremental iterative Newton-Raphson solution lead to out of balance force will be vanish. However, the Newton-Raphson technique fails in the neighborhood of critical points as the stiffness matrix approaches the singularity. Modified Newton-Raphson procedure works fine as long as the problem is displacement controlled one responded is not a snapback type. For several problems, the usual iterative procedures suggest that no equilibrium states exist in the post-peak regime, which, however, is not true. Moreover, most real problems are load controlled and cannot be made displacement controlled directly. The nonlinear solution technique and overall nonlinear solution strategy to be adopted are the most important for nonlinear pre- and post-yielding analyses of concrete members. The displacement control solution producers like Crisfield's arc-length method, Riks' arc-length method and modified Riks' arc-length method can be used to obtain the complete inelastic response of structure. Most of the inelastic analysis of structure bring singular vale of the diagonal element of stiffness matrix and unstable region will be appear due to the changing in the sign from positive to zero or negative value. The strain-softening is a poplar case for negative stiffness and unstable condition (Fig. 1) if traditional nonlinear solution techniques of Newton's family are used in the inelastic analysis of structures. 


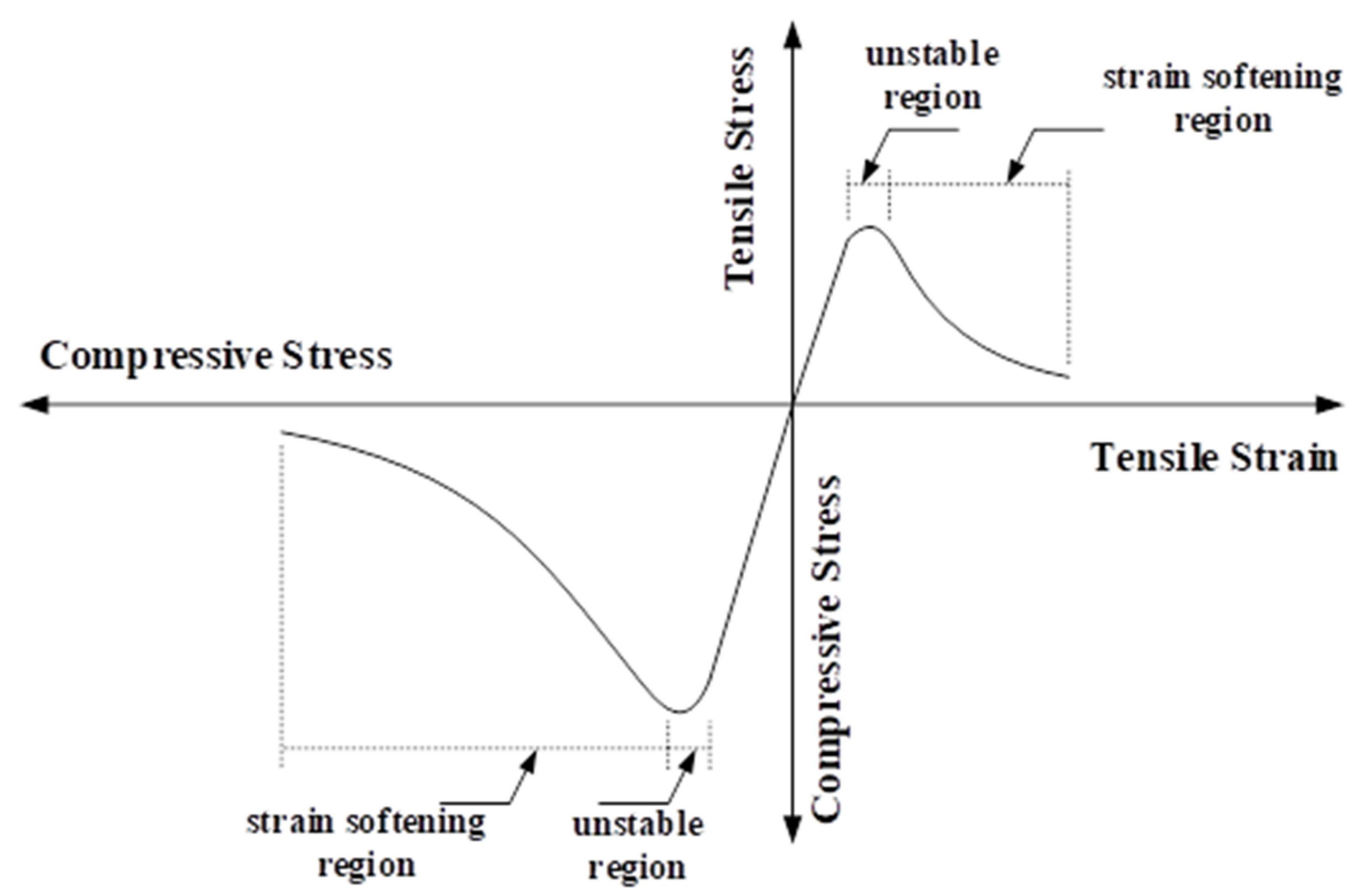

Fig. 1. Typical stress-strain relationship of concrete with unstable and strain-softening regions.

The Newton methods will be failed due infinite loop in the degradation part of the loaddeflection curve and the solution will be terminated due the negative or zero value in the stiffness matrix, to solve this problem the arc-length method is need to switched and used. This benefit is reached when the arc-length technique is used because both the load and displacement parameters are kept variable in the arc-length method, as the control equation in the arc-length method is written in functions of arc-length $(\Delta \mathrm{l})$ which is the function of both of these two terms [Crisfield (1991)]. Several numerical difficulties are observed in the nonlinear solution process even if the arc-length method is used. Load factor may become negative or deterioration in the solution procedure may occur. The most power techniques that is famlliar with the finite element analysis to pass unstable region of the stress-strain curve and also in the strain-softening region is Crisfield's strategy that can be used to avoid the numerical complexities related to the nonlinear post-yielding analyses of structures.

\section{DEVELOPMENTS IN AUTOMATIC LOAD-DISPLACEMENT CONSTRAINTS}

Several strategies have been developed to overcome the difficulties at the limit stage and to trace the response beyond this stage. The most commonly used techniques for this issue are discussed as follows. Special emphasis is laid on the arc-length or indirect displacement control technique, which enables to trace not only the post-critical response but is effective through the entire range of loading. 


\subsection{Artificial Spring Method}

This method was developed by Sharifi and Popov $(1971,1973)$. This method is valid when the single spring is use for a simple structure and load pattern and the method becomes mor complicated from a mathematical viewpoint when multiple springs are added. This method was developed for frames by Wright and Gaylord (1968). The observation of the snap in the problem is the main object of this technique that is transformed into one with a positive definite characteristic if linear and artificial springs are added to the system. Regarding Fig. 2, the vector of external loads $G^{i}$ of the modified system is decomposed into the real load vector ${ }^{\mathrm{i}} \mathrm{P}$ and the part resisted by the springs ${ }^{\mathrm{i}}$ giving

$$
G^{i}=P^{i}+f^{i}
$$

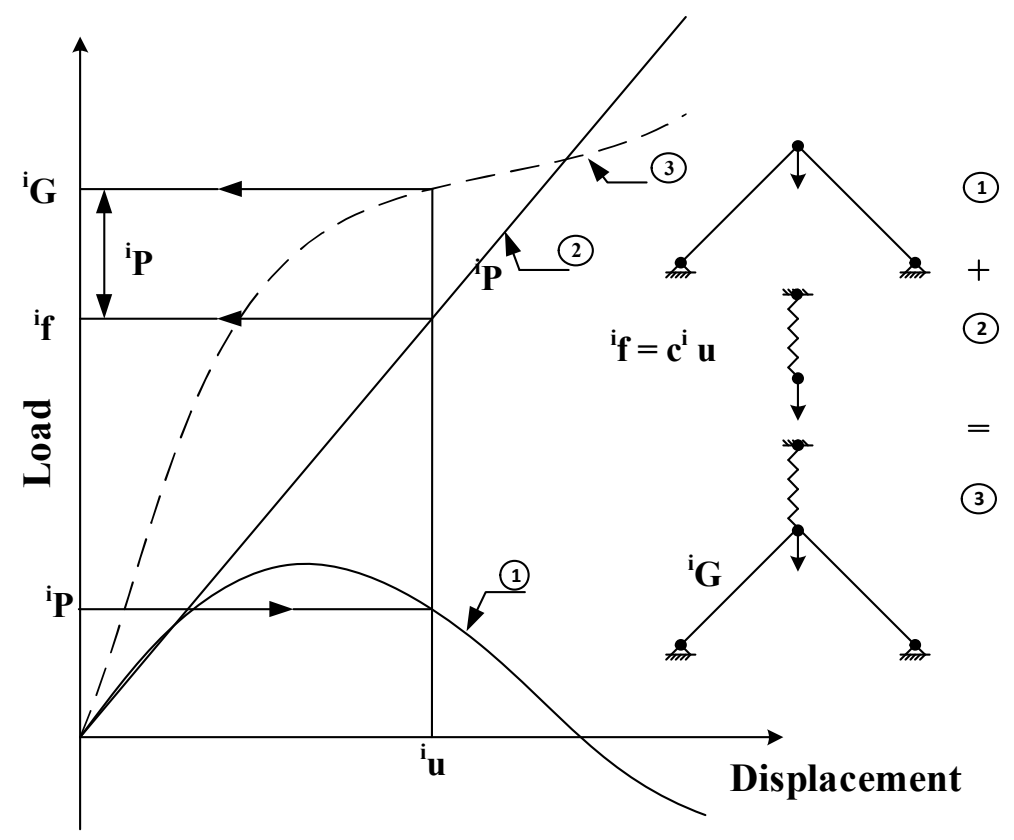

Fig. 2 Artificial spring method (Wright and Gaylord (1968)).

To retain the desired ratio of the specified loads it is required that all the components of the real load can be obtained by one common "load reduction factor" $\gamma^{\mathrm{i}}$

$$
P^{i}=\gamma^{i} G^{i}
$$

in other words, all components at configuration $\mathrm{i}$ have the same ratio

$$
\frac{f_{k}^{i}}{G_{k}^{i}}=1-\gamma^{i}
$$

It follows that springs have to be attached to all loaded degrees of freedom and all spring stiffness are coupled. Numerical experience shows that the method is successful only in a real 
snap-through problem (Ramm, 1980). The method is not recommended for structures with a local buckling or when a tendency to bifurcation is present.

\subsection{Suppressing Equilibrium Iterations (The Current Stiffness Method)}

The current stiffness method "The Current Stiffness parameter" introduced by Bergan (1978, 1980), it discussed how to deal with the limit points and the bifurcation encountered in structural response. The simplest way to avoid this difficulty is to suppress the iterations in the critical zone. The so-called current stiffness parameter measures the overall stiffness in the incremental direction in the solution space. This current stiffness parameter is scaled by the initial stiffness to achieve a non-dimensional quantity. For proportional loading, the current stiffness parameter may be expressed as

$$
S_{p}=\left\{\frac{\delta \lambda^{(i)}}{\delta \lambda^{(1)}}\right\}^{2} \frac{\delta u^{(1)^{T}} K^{(1)} \delta u^{(1)}}{\delta u^{(i)^{T}} K^{(i)} \delta u^{(i)}}
$$

where

$S_{p}=$ Current stiffness parameter Indices 1 and $\mathrm{i}$ refer to the first and the $i^{\text {th }}$ loading step respectively, $K=$ Tangent stiffness, $\delta u^{(i)}=$ Incremental displacement produced by $K^{(i)}$ for load increment., $\lambda^{(i)}=$ Load increment.

The unity value is use in order to check the system becomes softer (less than unit) or stiffer (greater than unit) as compared with initial state. The current stiffness parameter becomes zero with high property and pass peak point. Hence an observed low value of $\mathrm{S}_{\mathrm{p}}$ may be taken as a warning of the approaching singularity, and the equilibrium iterations should be suppressed when

$$
\left|S_{p}\right|<\bar{S}_{p}
$$

Normally the threshold value $\bar{S}_{p}$ is chosen between 0.04 to 0.12 (Bergan, 1982).

\subsection{Displacement Control Method}

The displacement control method will be used to obtain the load-deflection curve with the degradation zone. The method is illustrated in Fig. 3. In this algorithm, one component $(l)$ of the incremental displacement vector is considered to have a preselected value, i.e.,

$$
u^{(i)}|l=\hat{u}| l
$$

The displacement control method was originally introduced to avoid singularity at the critical point. The procedure was first introduced by Argyris (1967) and has subsequently been 
modified by Sabir and Lock (1972), Batoz and Dhatt (1979), and Ise (1982). Argyris (1967) used method that released set of nonsymmetrical equations. The displacement control method fails whenever the structure snaps back from a higher load level to a lower one. The displacement control method needs idea of the structure failure mode. Batoz and Dhatt (1979) described the computational procedure to be followed to overcome this problem. In the algorithm, the tangent stiffness matrix, $K$, is used to compute $\Delta u_{r}$, the incremental displacement produce from residual forces, $R^{(i-1)}$ at the end of $(i-1)$ th iteration and also $\Delta u_{e}$ that due to an incremental external load increment, $p$, i.e.,

$$
\begin{gathered}
\Delta u_{r}^{(i)}=K^{-1} R^{(i-1)} \\
\Delta u_{e}^{(i)}=K^{-1} p \\
R(u, \lambda)=p_{i}(u)-\lambda p_{e f} \\
\lambda=\lambda_{o}+\delta \lambda^{(i-1)}
\end{gathered}
$$

where $p_{i}$ is the internal forces which are functions of the displacements, $\mathrm{u}$, the vector $p_{e f}$ is a "fixed external loading vector" and the scalar $\lambda$ is a "load-level parameter" that multiplies $p_{e f}$ and $i$ is the iteration number. Since the actual incremental displacement vector, $\Delta u$, at iteration $\mathrm{i}$ is then given by

$$
\Delta u^{(i)}=\Delta u_{r}^{(i)}+\Delta \lambda^{(i)} \Delta u_{e}^{(i)}
$$

from (6) into (10) it follows that the change in the proportional load parameter is obtained as;

$$
\begin{array}{cr}
\Delta \lambda^{(i)}=\frac{\hat{u}\left|l-\Delta u_{r}^{(1)}\right| l}{\Delta u_{e}^{(i)} \mid l} & \text { for iteration (1) } \\
\Delta \lambda^{(i)}=-\frac{\Delta u_{r}^{(i)} \mid l}{\Delta u_{e}^{(i)} \mid l} & \text { for iteration (i), } \mathrm{i}>1
\end{array}
$$




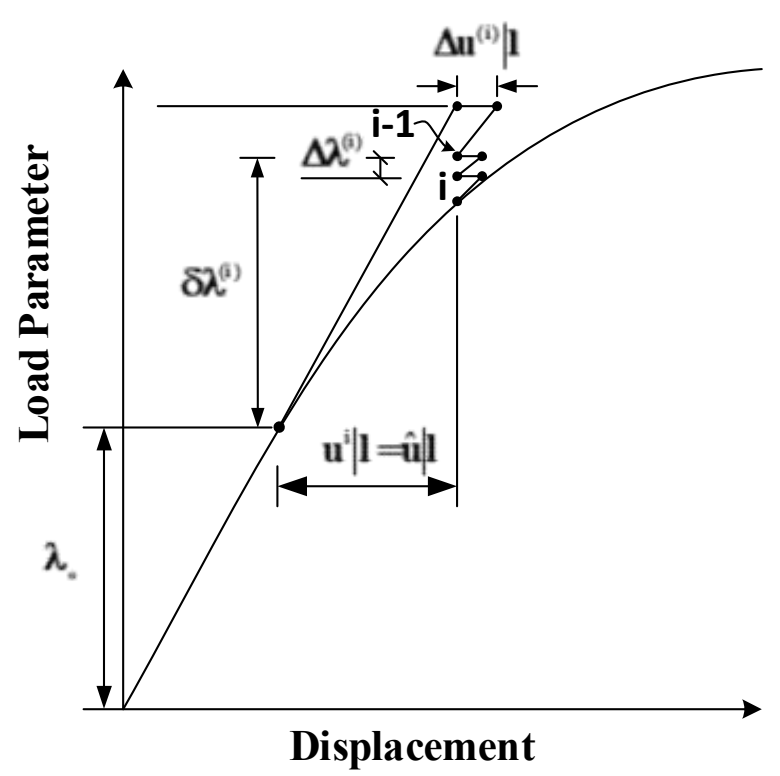

Fig. 3 Displacement control method (Batoz and Dhatt (1979).

The advantage of the above technique is that it can get the increment at the starting point of the iteration, the further sequence iteration are executed in the same procedure but $\delta$ value assign as zero. The iteration procedure will be reach to convergence at each nodal displacement. The above procedure fails for a case in which a reversal in the controlling displacement occurs (Simo at el. (1984). The structure has a snap-through or snap-back response, the use of this method leads to error.

\subsection{Arc-Length Method}

The arc-length method is one of the solution process used to trace equilibrium paths beyond limit points, the displacement will not change during one increment. This method is not only automatic but also improves the iterative performance away from limit points. The main objective of the arc-length method is the freedom to change load factor within a step i.e. within the iteration cycle. There are several methods for arc-length to obtain incremental load factor within one step. The method was originally proposed by Wempner (1971) and Riks $(1972,1979)$ and, subsequently modified by Ramm (1980) and Crisfield $(1981,1983)$ and by several others. The finite element method is familiar with the above procdure and is easy to implement and integrate with it. The arc-length method development procedures are described below;

\subsubsection{Riks orthogonal plane arc-length control}


Fig. 4 shows the use of Riks procedure to the solution of a one-dimensional problem. The control equation for problem having displacement variable $(\mathrm{N})$ are described as follows [(Wempner, 1971) and (Riks (1979)];

$$
\Delta u^{T} \Delta u+\Delta \lambda^{2} p^{T} p=\Delta l^{2}
$$

$\Delta u$ is vector of the incremental displacement vector and $\mathrm{p}$ is vector of the total external load. In every iteration, the satisfaction of the introduced constraint is required. The scalar $(\lambda)$ is called a loading parameter whereas $\Delta l$ is a recommended scalar, which fixes the length of the increase in $(\mathrm{N}+1)$ dimensional space. The $\mathrm{N}$ equilibrium equations involve the $(\mathrm{N}+1)$ unknowns consisting of $\mathrm{N}$ number of displacements, $u$ and $\lambda$, and can be written in the form:

$$
p_{\text {int. }}(u, \lambda)-\lambda p_{\text {ext. }}=0
$$

(The external load vector $p_{\text {ext. }}$ is normally referred to as $\mathrm{p}$ ).

The control equation devastated the banded and symmetry of the stiffness matrix when the incremental stiffness is added. A two-step technique can be used to solve this problem (Batoz and Dhatt (1979), Meek and Tan (1984). Riks-Wempner's approach is lead to changing of the iterative will be orthogonal to the predictor solution.

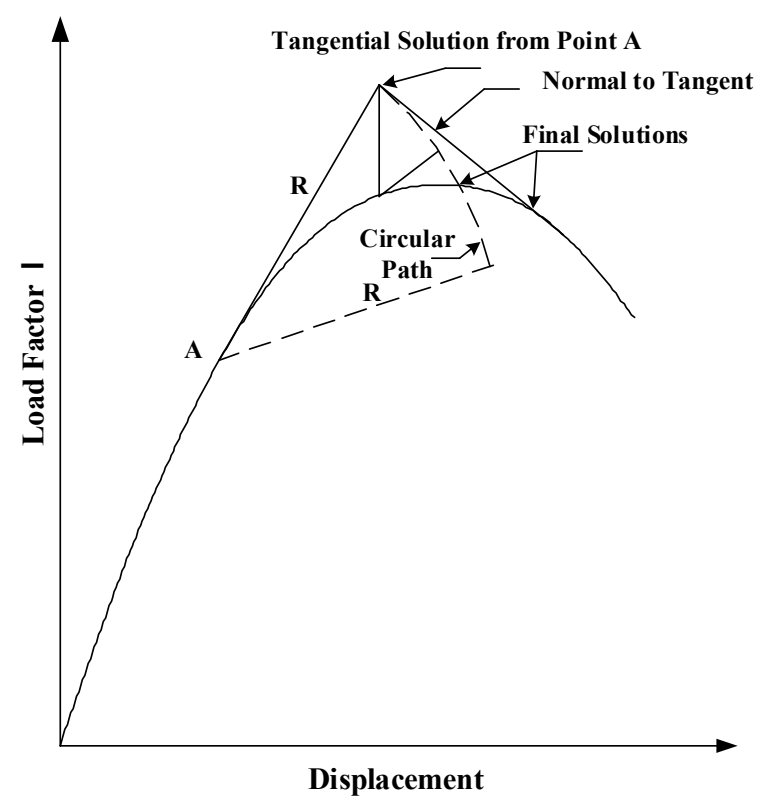

Fig. 4. Riks arc-length with Newton-Raphson technique (Riks, 1979).

\subsubsection{Cylindrical arc-length control of Crisfield}

The Riks technique can not implemente with standard finite element method with modified Newton Raphson method because of the banded nature of stiffness matrix of the lead to destroyed of the control equation [Crisfield (1981)]. In the loading increment with fixed 
incremental length $(\Delta \mathrm{l})$, Crisfield (1981) proposed the following fully quadratic construed equation that is depend on prdeditor step and correct step:

$$
\Delta u^{t} \Delta u=\Delta l^{2}
$$

It is shown that this constraint equation is numerically more consistent than the equation (14). The cylindrical arc-length method is shown in Fig. 5. The converge cannot be reached in the Crisfield method therefore another technique is needed to use. Hellweg and Crisfield (1998) suggested a different algorithm to choose the exact root for the cylindrical arc-length technique by selecting the root with the minimum residual norm.

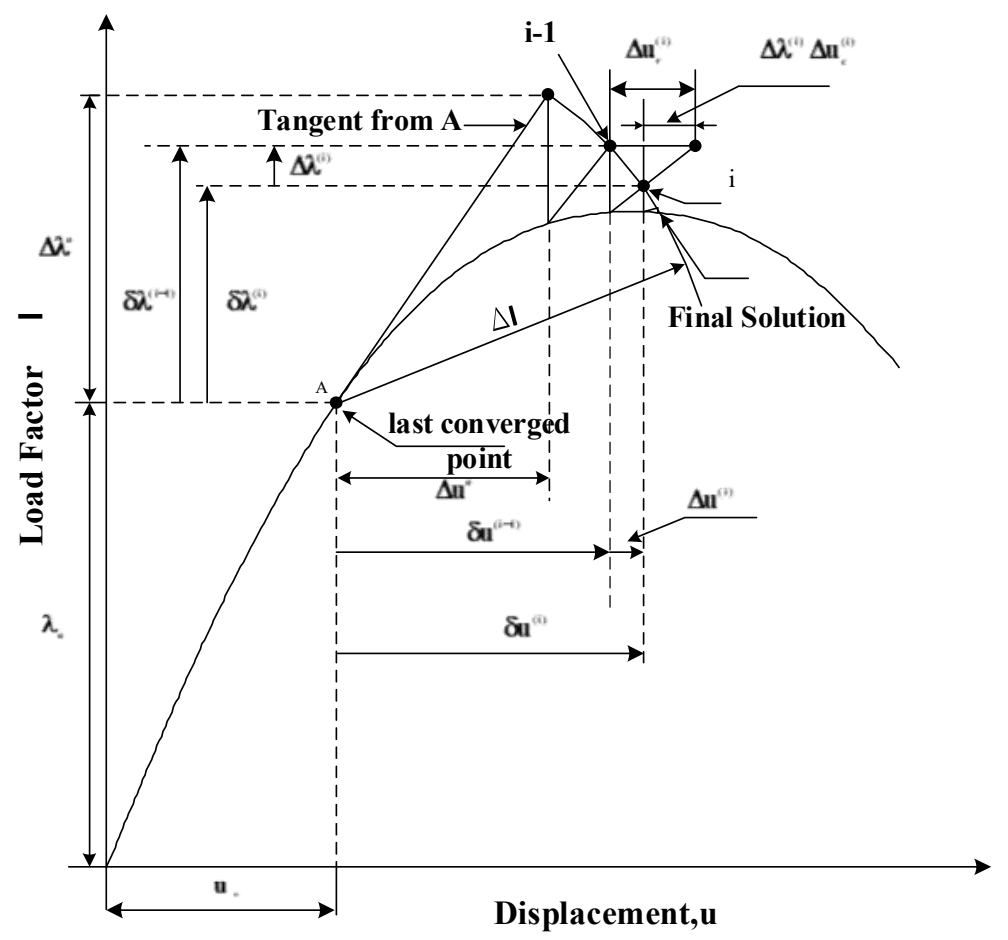

Fig. 5. Crisfield arc-length method (Hellweg and Crisfield, 1998).

Crisfield equation can be repeat on spherical constant arc-length method and cylindrical constant arc-length method in the displacement-load hyperspace.

\subsubsection{Ramm updated orthogonal arc-length control}

A modified "constant arc-length" presented by Ramm (1982) as strategy of Riks and Wempner. To overcome the shortcoming of Riks procedure, Ramm $(1980,1982)$ presented a simplified displacement control procedure, wherein the iteration path follows a "plane" normal to the tangent and it uses a new tangent with the "normal plane" as the solution progresses as shown in Fig. 6. The constraint equation can be solved without equilibrium equation. The value load increment for Ramm is given by the following expression; 


$$
\Delta \lambda^{(i)}=\frac{-\delta u^{(i-1) T} \Delta u_{r}^{(i)}}{\delta \lambda^{(i-1)}+\delta u^{(i-1) T} \Delta u_{e}^{(i)}}
$$

where $\Delta \mathrm{u}_{\mathrm{r}}^{(\mathrm{i})}$ and $\Delta \mathrm{u}_{\mathrm{e}}^{(\mathrm{i})}$ are defined in equation (7 and 8) and $\delta u^{(i-1) t h}$ incremental displacement up to the (i-1)th iteration. In this technique, the "iterative change" $(\delta u, \delta \lambda)$ is orthogonal to the "secant change" $\left(\Delta u_{o}, \Delta \lambda_{o}\right)$.

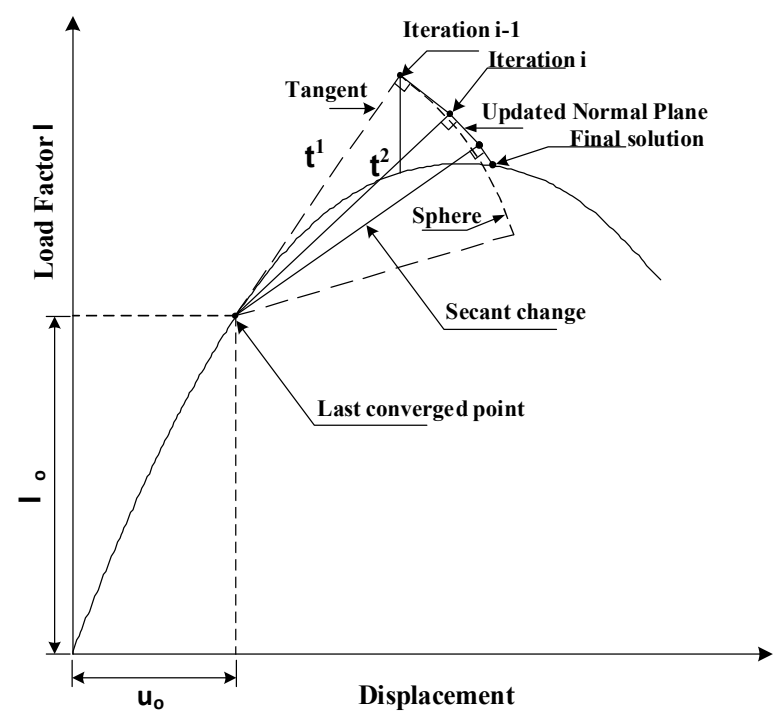

Fig. 6. Updated normal plane method "Ramm Method" (Ramm (1980, 1982).

\subsubsection{Fried orthogonal trajectory arc-length control}

Fried (1984) presented an alternative technique to Riks-Wempner-Crisfield iterative correction. Its symmetry concerning the displacement and the load guarantees achievement around passing peak points as well as turning points. It was considered the single, implicit equilibrium curve in mathematical form:

$$
r(x, \lambda)=0
$$

where $\mathrm{x}$ is define as the displacement and $\lambda$ is the load. The nonlinear $\mathrm{r}(\mathrm{x}, \lambda)$ is need to solve with iterative methods. The following two main points are required to fix in the solution procedure (i) how to advance from an recognized equilibrium $\left(\mathrm{x}_{0}, \lambda_{0}\right)$ to a next guess $\left(\mathrm{x}_{1}, \lambda_{1}\right)$ and (ii) what corrective method to use if $\mathrm{r}\left(\mathrm{x}_{1}, \lambda_{1}\right)$ is not acceptable. Let $\left(\mathrm{x}_{\mathrm{n}}, \lambda_{\mathrm{n}}\right)$ be a point in the $(\mathrm{x}, \lambda)$ plane not necessarily satisfying the equilibrium equation, $\mathrm{r}\left(\mathrm{x}_{\mathrm{n}}, \lambda_{\mathrm{n}}\right) \neq 0$. Let further $\delta \mathrm{x}$ $=\mathrm{x}_{\mathrm{n}+1}-\mathrm{x}_{\mathrm{n}}$ and $\delta \lambda=\lambda_{\mathrm{n}+1}-\lambda_{\mathrm{n}}$ denote the displacement and load corrections. Linearization of $\mathrm{r}\left(\mathrm{x}_{\mathrm{n}}+\delta \mathrm{x}, \lambda_{\mathrm{n}}+\delta \lambda\right)=0$ yields

$$
r_{n}+r_{n}^{\prime} \delta x+\dot{r}_{n} \delta \lambda=0
$$


where $\mathrm{r}^{\prime}=\frac{\partial \mathrm{r}}{\partial \mathrm{x}}$ (is stiffness matrix) and $\dot{\mathrm{r}}=\frac{\partial \mathrm{r}}{\partial \lambda}$ (is the load vector).

It was presented an improvement technique whereby the first guess $\left(\mathrm{x}_{1}, \lambda_{1}\right)$ is frequentative changed on an orthogonal line to the equilibrium curve, but with no investigative expression for it and without the contribution of the formerly calculated $\left(\mathrm{x}_{0}, \lambda_{0}\right)$. A comparative study of mathematical equations for incremental displacement and incremental load for the RiksWempner method, Crisfield method, and Fried method are given in Table 1.

Table 1. Mathematical expressions for different arc-length methods.

\begin{tabular}{ccc}
\hline Methods & Incremental load factor & Incremental displacement factor \\
\hline Riks-Wempner method & $\Delta \lambda=-\frac{r \dot{r}_{o}}{r^{\prime} r_{o}^{\prime}+\dot{r} \dot{r}_{o}}$ & $\Delta x=-\frac{r r_{o}^{\prime}}{r^{\prime} r_{o}^{\prime}+\dot{r} \dot{r}_{o}}$ \\
Crisfield method & $\Delta \lambda=-\frac{r}{\dot{r}-r^{\prime} \frac{\lambda-\lambda_{o}}{x-x_{o}}}$ & $\Delta x=\frac{r}{r^{\prime}-\dot{r} \frac{x-x_{o}}{\lambda-\lambda_{o}}}$ \\
Fried method & $\Delta \lambda=-\frac{r \dot{r}}{r^{\prime 2}+\dot{r}^{2}}$ & $\Delta x=-\frac{r r^{\prime}}{r^{\prime 2}+\dot{r}^{2}}$ \\
\hline
\end{tabular}

\subsubsection{Consistently linearized spherical hyperplane}

Schwiezerhof and Wriggers (1986) also advocated a linearised form of arc-length methods. It was used different constraint for the arc-length in every iteration cycle these by meaning variable constraint within an increment. This method is depend on solution with two step and still further for corrector steps two alternative proposals have been recommended depending upon the choice of the constraint. In the first alternative, The iteration solution on constant normal (hyper) plane for the first iteration and updated normal planes for subsequent iteration and whereas in the second alternative the iteration solution on spherical hyper planes as shown in Fig. 7. Further, it is improved the performance of arc-length method combined with the modified Newton method by employing line search schemes where diverges is occurs. The increment load factors as per Schwiezerhof and Wriggers for iteration on normal planes and spherical hyper plane respectively are given under:

$$
\begin{gathered}
\Delta \lambda^{(i)}=-\frac{f^{(i-1)}+\delta u^{(i-1) T} \Delta u_{r}^{(i)}}{\beta^{2} \delta \lambda^{(-i)}+\delta u^{(i-1) T} \Delta u_{e}^{(i)}} \\
\Delta \lambda^{(i)}=-\frac{f^{(i-1)}\left(f^{(i-1)}+s\right)+\delta u^{(i-1) T} \Delta u_{r}^{(i)}}{\beta^{2} \delta \lambda^{(i-1)}+\delta u^{(i-1) T} \Delta u_{e}^{(i)}}
\end{gathered}
$$


where $\Delta \mathrm{u}_{\mathrm{r}}^{(\mathrm{i})}$ and $\Delta \mathrm{u}_{\mathrm{e}}^{(\mathrm{i})}$ are defined in equations (7 and 8), where $(f)$ is the residual for the orthogonality expression, $(s)$ is the desired length and $\beta$ is the scaling factor.

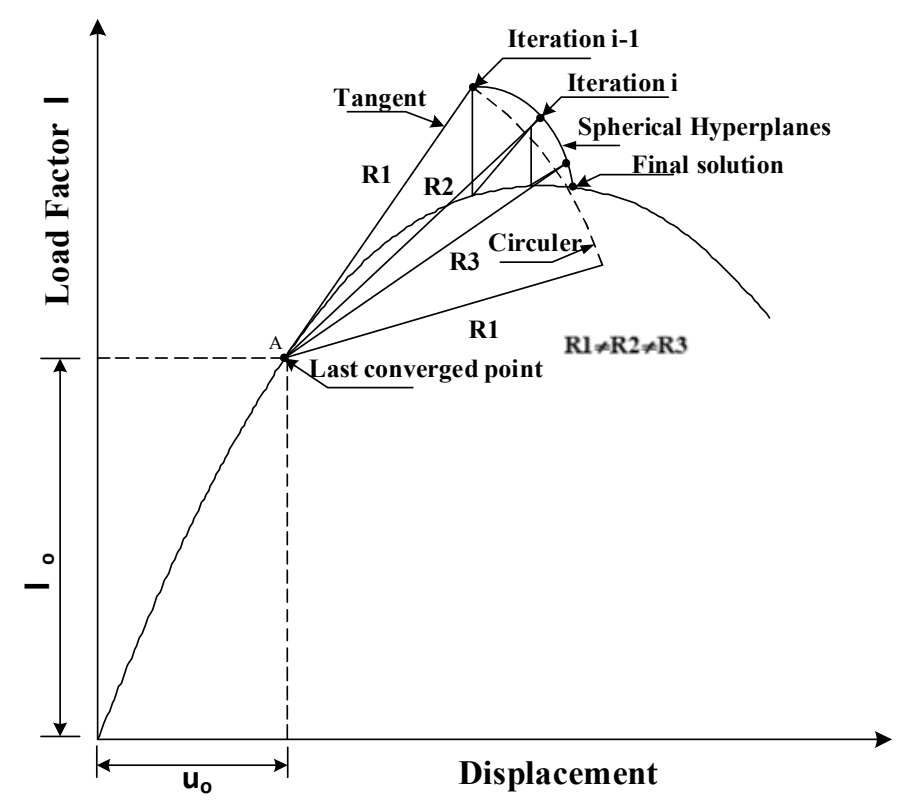

Fig. 7. Spherical hyperplanes method (Schwiezerhof and Wriggers, 1986).

\subsubsection{Improved arc-length orthogonality methods}

The improved arc-length method presented by Forde and Stiemer (1987), it was considered a linearized form of arc-length. The main principal of the improvement is orthogonality principles as shown in Fig. 8, that shows new procedure obtain the relation between existing path and the proposed path. The general formulation leads to the simplified procedure that it results gives similar results to Crisfield's explicit iteration procedure (1981). Iteration procedures such as normal and updated normal plane (Ramm, 1980), explicit spherical (Crisfield, 1981) and consistently linearized spherical hyper plane (Schweizerhof and Wriggers, 1986), all follow from the results of this general method under some specific conditions. Forde and Stiemer proposed a general expression for load increment as:

$$
\Delta \lambda^{(i)}=\frac{f^{(i-1)}-\delta u^{(i-1) T} \Delta u_{r}^{(i)}}{\beta^{2} \delta \lambda^{(i-1)}+\delta u^{(i-1) T} \Delta u_{e}^{(i)}}
$$

where $\beta$ is the scaling factor $\Delta \mathrm{u}_{\mathrm{r}}^{(\mathrm{i})}$ and $\Delta \mathrm{u}_{\mathrm{e}}^{(\mathrm{i})}$ are defined in equation $(7$ and 8$)$. The load increment $(\Delta \lambda)$ is the same value as that obtained by Crisfield (1981). Forde and Stiemer used $\mathrm{f}^{(\mathrm{i})} \equiv 0$, and the tangent $\mathrm{t}^{(\mathrm{i})}$ and update $\mathrm{n}^{(\mathrm{i})}$ are mutually normal at each iteration step, as given below. 


$$
\Delta \lambda^{(i)}=\frac{-\delta u^{(i-1) T} \Delta u_{r}^{(i)}}{\beta^{2} \delta \lambda^{(i-1)}+\delta u^{(i-1) T} \Delta u_{e}^{(i)}}
$$

When divergence observed from the arc, the simple corrections needs to be proceeds to the residual. The residual for the orthogonality expression can be obtained from the project of the difference between the length of the recent tangent vector and the chosen length onto the recent tangent vector:

$$
t^{(i-1)} r^{(i-1)}=-\left|t^{(i-1)}\right|\left|r^{(i-1)}\right|=-t^{(i-1)}\left(t^{(i-1)}-s\right)=f^{(i-1)}
$$

The linearized arc-length can be updated by substitution of this residual or load factor on the spherical hyperplane as obtained by Schweizerhof and Wriggers (1986) and given below;

$$
\Delta \lambda^{(i)}=\frac{-t^{(i-1)}\left(t^{(i-1)}-s\right)-\delta u^{(i-1) T} \Delta u_{r}^{(i)}}{\beta^{2} \delta \lambda^{(i-1)}+\delta u^{(i-1) T} \Delta u_{e}^{(i)}}
$$

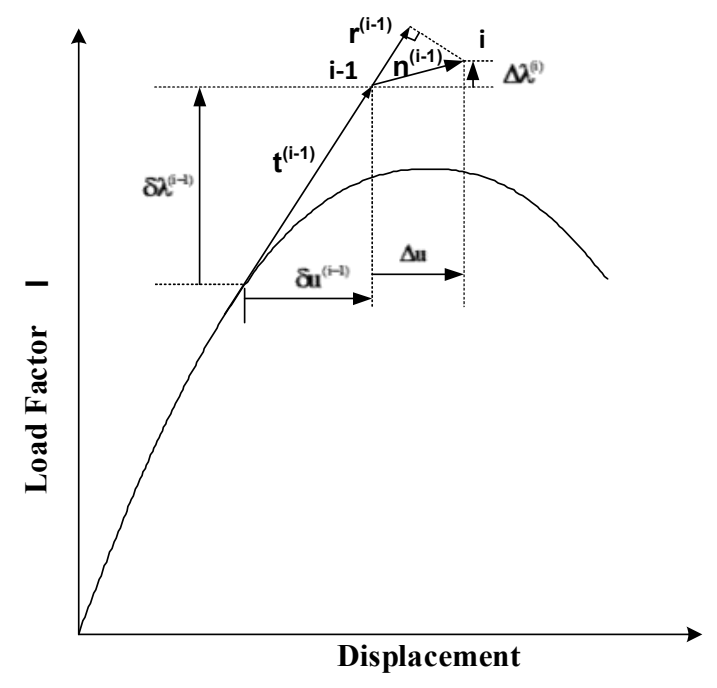

Fig. 8. Orthogonality relationship (Forde and Stiemer (1987).

\subsubsection{More development in the arc-length method}

The arc-length method suggested by Al-Rasby (1991) with two diagonal scaling matrix to determine of non-dimensional vectors. This method is nearly comparable to Forde and Stiemer (1987) except for the difference in the diagonal scaling matrices. The incremental load factor is given below.

$$
\Delta \lambda^{(i)}=\frac{R^{(i)}-U^{(i) T} V_{s} \delta^{(i)}}{\delta \lambda^{(i)} p_{e f}^{T} W_{s} p_{e f}+U^{(i) T} V_{s} \delta^{(i)}}
$$

where $p_{\text {ef }}$ reference load factor, $U^{(i)}$ incremental displacement vector, $\delta^{(i)}$ iterative displacement vector for iteration $\mathrm{i}, \mathrm{V}_{\mathrm{s}}$ is the defining of displacement scaling diagonal matrix, 
and $\mathrm{W}_{\mathrm{s}}$ is the defining of load scaling diagonal matrix. By changing the residual $\mathrm{R}$, all of the known arc-length methods can be evolved out of this i.e. with the suitable variant of $\mathrm{V}_{\mathrm{s}}$ and $\mathrm{W}_{\mathrm{s}}$, load control, and displacement control, all previously described methods reduce to particular cases of this technique. It was suggested four types of difference of the scaling diagonal matrices. Lam and Morley (1992) presented a new solution for arc-length to deal with the problem of complex roots to the quadratic equation.

Fafard and Massicotte (1993) suggested a method called the modified Crisfield-Ramm method that gets the benefits of the two-parent method (Crisfield, 1981) and (Ramm, 1981). the control equation proposed by Ramm (1981) was used by Fafard and Massicotte (1993) and used an changed hyper plane procedure to estimate arc-length as follows

$$
\begin{gathered}
\Delta \lambda=-\frac{\delta u^{(i-1) T} \Delta u_{r}^{i}}{\delta u^{(i-1) T} \Delta u_{e}^{i}} \\
\delta u^{(i)}=\delta u^{(i-1)}+\Delta u^{(i)}
\end{gathered}
$$

where $\delta u^{(i)}$ is represented the incremental displacement up to the ith iteration. Iterative displacement, $\Delta \mathrm{u}^{\text {(i) }}$ is calculated by using equation (11). The reduction of computed displacement is suggested as follow;

$$
\left\|\delta u^{i}\right\|=\Delta l=\alpha\left\|\delta u^{i}\right\|
$$

which gives; $\alpha=\frac{\Delta l}{\left\|\delta i^{i}\right\|}$

The desired incremental displacement is given as follow:

$$
\delta u^{i}=\alpha \delta u^{i-1}+\alpha \Delta u
$$

The main advantage of this method that is not prompt any convergence problem and the response of the structure is easier to obtain near the limit point.

Besides Fafard and Massicotte (1993) provided the geometrical demonstration of arc-length methods introduced by Crisfield and Ramm, which assistances to envision the method geometrically. A different procedure of which consistent linearization of the arc-length method was suggested by Carrera (1994), that improved Riks-Wempner and Ramm's methods, as explained under:

a) Riks-Wempner-Tangent Method: In the first proposed method Riks-Wempner-Tangent consists of a further improvement of the Ramm method. The incremental load factor from this method is computed as under; 


$$
\Delta \lambda^{(i)}=-\frac{\delta u^{(i-1) T} \Delta u_{r}^{(i)}}{\delta \lambda_{r}^{(i-1)} p_{e f}^{T} p_{e f}+\delta u^{(i-1) T} \Delta u_{e}^{(i)}}
$$

where $p_{\text {ef }}$ is a fixed reference load vector, $\delta \lambda_{\mathrm{r}}$ is the incremental load parameter related to the residual force. At $\mathrm{i}=1$ i.e. in the first iteration the method works as the Riks-WempnerMethod. The work tangent takes sense when the tangent operator is changed for each iteration.

b) Riks-Wempner-Normal Method: The incremental load factor arises from this method is:

$$
\Delta \lambda^{(i)}=-\frac{\delta u^{(i-1) T} \Delta u_{r}^{(i)}}{\delta \lambda^{(i-1)} p_{e f}^{T} p_{e f}+\delta u^{(i-1) T} \Delta u_{e}^{(i)}}
$$

at the $\mathrm{i}=1$-iteration as the Riks-Wempner- Method. Where $\Delta \mathrm{u}_{\mathrm{r}}^{(\mathrm{i})}$ and $\Delta \mathrm{u}_{\mathrm{e}}^{(\mathrm{i})}$ are defined in equations (7 and 8).

c) Determination of Roots: the new procedure to select the suitable root of a nonlinear constrained equation proposed by Carrera (1994). The computation of linear solution for the available date is needed to use. After that, the calculation of angle is required to get the proper root.

Zhou and Murray (1994) presented a modified arc-length constraint equation to avoid divergence of the technique coming because of the complex root. Relaxation factor, $\beta$, is the main principle of this method, which represented a fraction of the unbalanced force. The range of relaxation factor is from 0 to 1.0 when $\beta$ is set to be one the results become identical to the traditional arc-length. A physical meaning of the relaxation factor $\beta$ is a fraction of the unbalanced forces. The fraction force is used in iterations instead of full unbalanced force same as other iteration procedure. Consequently, the number of iteration may be increased, such that the stability of the iterative process can be improved and the probability of failure of the solution can be diminished. The proper value of factor $\beta$ always ensures positive discriminant of quadratic equation, to yield the real roots. The discriminant of the quadratic equation assigning by the value greater than or equal to zero can realize acceptable range of relaxation factor $(\beta)$, the quadratic equation will be in term of $(\beta)$. The $\beta$ values can be capture from new equation, the best $\beta$ value can be as $\left(0<\beta \leq \beta_{\max }\right)$ where $\beta_{\max }=\min \left(1, \beta_{2}\right)$, where $\beta_{2}$ is the second root of the quadratic equation, the batter efficiency can get when $\beta$ is increasing and the number of iteration is reduce. When the value of $\beta_{\max }$ is less than 0.01 , therefore the than the full Newton-Raphson method is recommended to use. 
Zhiliang (1994) presented a simple interpretation for the straight incremental/iterative solution, single specific displacement controlling method, and arc-length controlling method from their geometric characteristic, it was suggested to use load/ displacement control and cylindrical arc-length with zero incremental work norm. the two cylindrical shell problems with snap-back were used to verify the suggested method, the good obserivation validation results were obtained for derived method.

It has been decided that the control technique of the zero incremental displacement norm is more common and proficient. Kuo and Yang (1995) concluded that most incremental-iterative methods (including arc-length) for finding the post-buckling paths of the structure have unsuccessful to calculate a load-displacement response, and argued that it is because iterations were not performed in the proper direction for problems with multi-winding loops in the response.

Feng et al. (1996) suggested a new principle used in the analyst stage of the arc-length method, which may avoid false travel path of the arc-length method due to incongruous initial guesses. It was proposed a formulation for computation of first load increment, built on the method of the sign of the determinant of the current stiffness matrix. The new standard can be simply combined within both conventional and iterative solvers. To choose the correct sign of load factor several criteria have been proposed using the derivative of the load parameter at the current iteration.

May and Duan (1997) found the inclusion of the displacements of the dominant nodes, which are associated with the failure zone, in the constraint equation leads to improvement of results in structures involving softening and material nonlinearity. The nonlinear behaviour of structure leads to the problem due to dominant node and any node can be change due to this nonlinearity. This is give imported conclusion that a problem in constant equation becomes dependent therefore this is disadvantage for this method, to solve this problem May and Duan (1997) proposed local arc-length technique limited by control equation, the iteration course is used for all steps. The plane element has following relative displacement vector with $\mathrm{n}$ degree of freedom:

$$
\Delta \delta=\left[\delta_{1}-\delta_{n}, \delta_{2}-\delta_{1}, \delta_{3}-\delta_{2}, \ldots \delta_{n}-\delta_{n-1}\right]^{T}
$$

where $\delta_{1}$ to $\delta_{\mathrm{n}}$ are the nodal displacements in specific element. The relationship between arclength and load factor are given as follow:

$$
\sum_{e=1}^{m}\left(\Delta \delta_{1}\right)_{e}^{T}\left(\Delta \delta_{i}\right)_{e}=\Delta l^{2}
$$




$$
\Delta \lambda_{\mathrm{i}}=\Delta \lambda_{1}-\frac{\sum_{\mathrm{e}=1}^{\mathrm{m}}\left(\Delta \mathrm{U}_{\mathrm{P}}\right)_{\mathrm{e}}^{\mathrm{T}}\left(\Delta \delta_{\mathrm{i}-1}+\Delta \mathrm{U}_{\mathrm{F}}\right)_{\mathrm{e}}}{\sqrt{\sum_{\mathrm{e}=1}^{\mathrm{m}}\left(\Delta \mathrm{U}_{\mathrm{P}}\right)_{\mathrm{e}}^{\mathrm{T}}\left(\Delta \mathrm{U}_{\mathrm{P}}\right)_{\mathrm{e}}}}
$$

in which

$$
\Delta \lambda_{1}=\frac{\Delta l}{\sqrt{\sum_{e=1}^{m}\left(\Delta U_{P}\right)_{e}^{T}\left(\Delta U_{P}\right)_{e}}}
$$

where the subscripts 1 and $i$ are the first and ith iteration, the total number control element represented by $(\mathrm{m})$. The control displacement in the element is defined as $U_{\mathrm{P}}$, the incremental displacement problem from unbalanced load is defined as $U_{F}$. The behavior of lowdisplacement of strain-softening material predicts successfully as given in the reported results. However, the definite method was useful to plain concrete elements and there was no sign of the use of the technique for compression softening, which is common and main in concrete structures. Teng and Luo (1998) suggested a change to the standing arc-length method according to the idea of added arc-length. Introducing a new parameter $\gamma$ does this modification

$$
\gamma\left(L_{i}\right)=\lambda_{i}-\lambda_{d}
$$

where $\lambda_{i}$ is the converged load-factor, $\lambda_{d}$ is the chosen load level and $\mathrm{Li}$ is the summation of arc-lengths up to the current increment. The next load step $\left(L_{d}\right)$ computes the desired arclength increment by making the accumulated arc-length $L_{d}$ satisfy the following equation.

$$
\gamma\left(L_{d}\right)=0
$$

Hence ; $L_{i+1}=L_{i}+l_{d}$

The quadratic and linear expression are suggested in Taylor expansion in order to capture the solution of the above equation. If the new arc-length achieved is greater than the one obtained from the usual technique then the arc-length from the usual technique is used and the following evaluation of arc-length according to the collected arc-length technique is completed in the next step. The arc-length technique is best tool and familiar with finite element analysis of structure to capture load-deflection path (Ahmed and Xiao-Zu (2004)).

\section{SCALING PARAMETER}


The applied load factor is the additional varable to arc-length technique during iteration procedure, the load-displacement curve declined or fold back can be explored. The load increase is governed by an additional control equation with the common form

$$
\Delta u^{T} \Delta u+A_{o} \Delta \lambda^{2} p^{T} p=\Delta l^{2}
$$

Where; $\Delta \mathrm{u}=$ the test increase in displacement vector at the $\mathrm{i}^{\text {th }}$ iteration, $A_{o}=$ a scalar parameter that governs the relative contributions of the displacement and load increments, $\Delta \lambda=$ the predicted load factor, $\mathrm{p}=$ the specified external load vector and $\Delta l=$ the specified arc-length for the current increment. When the scaling parameter $\left(A_{o}\right)$ equals zero the procedure will be reduced to "cylindrical arc-length procedure" which otherwise falls under the procedure becomes "spherical arc-length procedure". Crisfield (1981) and Ramm (1981,1982) indecently concluded that for particular problems involving a realistic number of variables, the loading terms (those involving $A_{o}$ ) had little effect and hence advocated setting $A_{o}$ to zero. As a result, the cylindrical constraint should be considered as "cylindrical" rather than "spherical". The scaling parameters proposed by Lam and Morley (1992) is:

$$
A_{o}=\frac{u_{o}{ }^{T} u_{o}}{\lambda_{o}{ }^{2}}
$$

where $u_{o}$ and $\lambda_{o}$ are the vectors of nodal displacement and last load factor at the last converged.

\section{CONCLUDING REMARKS}

A critical review of literature has been completed to recognize the difficulties faced in the calculation of whole load-displacement performance of structures and methods available to overcome the same. The only arc-length method has been reviewed which can be assimilated with the traditional finite element method. The use of arc-length algorithm can be used tis to apprehension the solution of the global stiffness matrix with ill-condition. From the above review, Crisfield's technique is found to be the best proficient one for the complete inelastic analysis of structure near limit point and adopted in the many research studies.

\section{REFERENCES}

Crisfield, M.A. (1991) 'Non-Linear Finite Element Analysis of Solids and Structures', Vol. 1, 1st ed., John Wiley \& Sons.

Sharifi, P. and Popov, E.P. (1971) 'Nonlinear buckling analysis of sandwich arches', Journal of the Engineering Mechanics Division, ASCE, 97,PP. 1397-1411. 
Sharifi, P. and Popov, E.P. (1973) 'Nonlinear finite element analysis of sandwich shells of revolution', AIAA Journal, 11, PP. 715-722.

Wright, E.W. and Gaylord, E.H. (1968) 'Analysis of unbraced multistoreyed steel rigid frames', Journal of the Structural Division, ASCE, 94, PP. 1143-1163.

Ramm, E., (1980) 'Strategies for tracing the nonlinear response near limit points', Proceeding Of the Europe-U.S. workshop 'nonlinear finite element analysis in structural mechanics', Edited By Wunderlich, W., Stein, E. and Bathe, -J., Ruhr-Universitat Bochum, Germany, July 28-31.

Bergan, P.G., Horrigmoe, G., Bråkeland, B., and Søreide, T.H. (1978) 'Solution techniques for non-linear finite element problems', International Journal for Numerical Methods in Engineering, 12, PP. 1677-1696.

Bergan, P.G (1980) 'Solution algorithms for nonlinear structural problems', Computers and Structures, Vol.12, No.4, PP. 497-509.

Bergan, P.G And Soreide, T.H. (1978) 'Solution of large displacement and instability problems using the current stiffness parameter', Proceedings Of The International Conference On Finite Elements In Nonlinear Mechanics, Stuttgart, Germany, August 30 To September 1, Editors: Pister, K. S., Reynolds, R. R., And Willam, K.

Bergan, P.G. (1982) 'Automated incremental-iterative solution methods in structural mechanics', In' Recent Advances In Non-Linear Computational Mechanics', Edited By Hinton, E., Owen, D.R.J. And Taylor, C., Pineridge Press Limited, Swansea, U.K.

Argyris, J.H. (1967) 'Continua and discontinua', In Proceedings Conference On 'Matrix Methods in Structural Engineering', Wright-Patterson Air.Force.Base, Ohio, PP.11-189.

Sabir, A.B., and Lock, A.C. (1972) 'The application of finite elements to large deflection geometrically nonlinear behaviour of cylindrical shells', in, 'Varaiational methods in engineering' Edited by Brebbia C.A. And Tottenham, H.,Southampton, University Press, 7/66-7/75.

Batoz, J.L., And Dhatt, G. (1979) 'Incremental displacement algorithms for nonlinear problems', International Journal for Numerical Methods in Engineering, 14, PP.1262-1267.

Ise, G. (1982) 'Analysis of eccentrically stiffened wide panels', In 'Recent advances in nonlinear computational mechanics', Edited By Hinton, E., Owen, D.R.J. And Taylor, C., Pineridge Press Limited, Swansea, U.K. 
Simo, J.C., Wriggers, P., Schweizerhof, K.H. and Taylor, R.L, (1984), "Finite Deformation Postbuckling Analysis Involving Inelasticity And Contact Constraints", In Proceedings Of The International Conference On "Innovative Methods For Nonlinear Problems", Edited By Liu, W.K, Belytschko, T. And Park, K.C., Pineridge Press International Limited, Swansea, U.K., Pp.365-387.

Wempner, G.A. (1971) 'Discrete Approximation Related To Nonlinear Theories of Solids', International Journal Solids and Structures, 7, PP. 1581-1599.

Riks, E. (1972) 'The application of Newtons' method to the problem of elastic stability', Journal of Applied Mechanics, ASME, 39, pp. 1060-1065.

Riks, E. (1979) 'An incremental approach to the solution of snapping and buckling problems', International Journal Solids and Structures, 15, pp. 529-551.

Crisifield, M.A., (1981) 'A Fast Incremental/Iterative Solution Procedure That Handles SnapThrough ', Computers and Structures, Vol.13, PP.55-62.

Crisifield, M.A.,(1983) 'An arc-length method including line searches and accelerations', International Journal for Numerical Methods in Engineering, 19, PP. 1269-1289.

Meek, J.L. and Tan, H.S. (1984) 'Geometrically nonlinear analysis of space frames by an incremental iterative technique', Computer Methods in Applied Mechanics and Engineering, 47, PP. 261-282.

Hellweg, H. B., and Crisfield, M.A., (1998), "A new arc-length method for handling sharp snap-backs", Computers and Structures, Vol.66, No.5, Pp.705-709.

Ramm, E. (1982) 'The Riks/Wempner approach - an extension of the displacement control method in nonlinear analysis', in 'Recent advances in non-linear computational mechanics', Edited by Hinton, E., Owen, D.R.J. And Taylor, C., Pineridge Press Limited, Swansea, U.K.

Fried, I., (1984), 'Orthogonal trajectory accession to the nonlinear equilibrium curve', Computer Methods in Applied Mechanics and Engineering, 47, Pp. 283-297.

Schweizerhof, K. and Wriggers, P. (1986) 'Consistent linearization for path following methods in nonlinear FE analysis', Computer Methods in Applied Mechanics and Engineering, 47, Pp. 283-297.

Forde, B.W.R. and Stiemer, S.F. (1987) 'Improved arc length orthogonality methods for nonlinear finite element analysis', Computers and Structures, Vol.27, Np.5, Pp. 625-630. 
Al-Rasby, S.N., (1991) 'Solution techniques in nonlinear structural analysis', Computer and Structures, Vol.40, PP. 985-993.

Lam, W.F. and Morley, C.T. (1992) 'Arc-length method for passing limit points in structural calculation', Journal of Structural Engineering, Vol.118, No.1, January.

Fafard, M., and Massicotte, B., (1993) 'Geometrical interpretation of the arc-length method', Computer and Structures, Vol.46, PP. 603-615.

Carrera, E., (1994), 'A study on arc-length type methods and their operation failures illustrated by a simple model', Computer and Structures, Vol.50, PP. 217-229.

Zhou, Z.L., and Murray, D.W., 1994, 'An incremental solution technique for unstable equilibrium paths of shell structures', Computer and Structures, Vol.55, PP. 749-759.

Zhiliang, F., (1994), 'A study of variable step-length incremental/iterative methods for nonlinear finite element equations', Computers and Structures Vol.52 (6), PP. 1269-1275

Kuo, S.R., Yang, Y.B., (1995), 'Tracing post buckling paths of structures containing multiloops', International Journal for Numerical Methods in Engineering, 38:4053- 4075.

Feng, Y.T., Peric, D., and Owen, D.R.J., 1996, 'A new criterion for determination of initial loading parameter in arc-length methods', Computer and Structures, Vol.58, PP. 479-485.

May, I.M., and Duan, Y., (1997), 'A local arc-length procedure for strain softening', Computer and Structures, Vol.64, PP. 297-303. 